\title{
Explosive instability of geostrophic vortices. Part 2: parametric instability
}

\author{
Xavier Carton ${ }^{1,{ }^{*}}$, Thomas Meunier ${ }^{2}$, Glenn R. Flierl ${ }^{3}$, Xavier Perrot ${ }^{2}$ and Mikhail A. Sokolovskiy ${ }^{4}$
}

\author{
${ }^{1}$ LPO, UMR 6523, UEB/UBO, Brest, France \\ 2 LPO, UEB/UBO, Brest, France \\ ${ }^{3}$ EAPS, MIT, Cambridge, MA, USA \\ ${ }^{4}$ Institute for Water Problems of the RAS, Moscow, Russia \\ *: Corresponding author : X. Carton, email address : xcarton@univ-brest.fr
}

\begin{abstract}
:
In a two-layer quasi-geostrophic model, a baroclinic vortex is submitted to a periodic forcing of its mean baroclinic azimuthal velocity. It is shown that parametric effects could stabilize a vortex which is baroclinically unstable in the absence of forcing. Conversely, parametric resonance can destabilize a baroclinically stable vortex, under conditions on the vortex parameters, on the ratio of layer thicknesses or on the forcing frequency.
\end{abstract}

Keywords: Quasi-geostrophic equations - Inviscid flows - Vortex stability - Parametric resonance

PACS 92.05.Bc - 92.10.ak - 92.10.ei - 47.15 - 47.20.Cq 


\section{Introduction}

In stratified rotating turbulence and in planetary fluids, vortices play an essential role in the transport of momentum, heat and tracers. The baroclinic instability of vortices has been studied with normal- mode perturbations ([1], [2], [3]). The nonlinear evolution of these vortices, perturbed with normal modes, usually leads to multipolar vortices. Part 1 of this paper has compared the properties of linear baroclinic instability of vortices with piecewiseconstant potential vorticity, perturbed with normal modes or with singular modes. In a timevarying flow, the resonance of baroclinically neutral waves and of the forcing can lead to parametric instability, in particular for parallel flows ([4]). Parametric instability has not yet been studied for vortex flows. Theoretical elements underlying this instability have been developed in ([5]). Here, we consider the parametric resonance of neutral waves with a periodic external forcing which modifies the baroclinic velocity of this vortex. We study the properties of this parametric instability.

\section{Model equations and parametric instability of an oscillating baroclinic vortex}

The forced two-layer quasi-geostrophic equations describe the evolution of layerwise potential vorticity

$\underline{\mathrm{d} q j}=E j(r, t), \quad q j=\nabla 2 \not j \mathrm{j}+F j(\not k-\not j)$

$\mathrm{d} t$ 
where $q_{j}$ is the layerwise potential vorticity (the subscripts $j=1,2$ denote upper and lower layers respectively, and $k=3-j), F_{j}$ are the layer coupling coefficients $\left(F_{j}=f_{0}^{2} / g^{\prime} H_{j}\right), H_{j}$ is layer thickness, and $H=H_{1}+H_{2}$. The internal deformation radius is $R_{d}=\sqrt{g^{\prime} H_{1} H_{2}} / f_{0} \sqrt{H}$ and $\gamma$ is its inverse. The term $E_{j}(r, t)$ is a time periodic forcing of the baroclinic mean flow.

If neutral waves traveling on the vortex periphery resonate with this forcing (like a pendulum whose axis of rotation oscillates vertically), they can be amplified via the process of parametric instability.

Part 1 of the paper has shown that the linear instability of a two-layer vortex with piecewiseconstant potential vorticity can be described in vertical modes by the equation $\partial_{t} X=A X$ with $X\left(\eta_{t}, \eta_{c}\right)$ and

$A=-i\left(\begin{array}{ll}a & b \\ c & d\end{array}\right)$

Calling $V_{t}(1), V_{c}(1)$ the barotropic and baroclinic velocities of the mean flow, at the vortex boundary, we have $a=l V_{t}(1)(1-1 / l), b=l V_{c}(1)\left(1-1 /\left(2 l I_{1}(\gamma) K_{1}(\gamma)\right), c=l V_{c}(1)\left(1-\left(I_{l}(\gamma) K_{l}(\gamma)\right) /\left(I_{1}(\gamma) K_{1}(\gamma)\right)\right.\right.$ and $d=l V_{t}(1)\left(1-2 I_{l}(\gamma) K_{l}(\gamma)\right)+\xi l V_{c}(1)\left(1-\left(I_{l}(\gamma) K_{l}(\gamma)\right) /\left(I_{1}(\gamma) K_{1}(\gamma)\right)\right.$, where $\xi=(1-\delta) / \sqrt{\delta}$ with $\delta=H_{1} / H_{2}$.

\section{Parametric instability near marginality of baroclinic instability}

We assume that the mean baroclinic velocity is close to that at marginality of baroclinic instability (called $V_{c}^{0}$ at the vortex boundary). The time periodic forcing adds a weak unsteady component to this velocity to allow parametric instability. The baroclinic azimuthal velocity at the vortex boundary is then written

$$
V_{c}(1)=V_{c}^{0}[1+\varepsilon h(t)]
$$

with $\varepsilon<<1, h(t)$ is a time periodic function. Under these conditions, the linearized dynamics matrix is

$$
A=A_{0}+\varepsilon h(t) A_{1}
$$

$A_{0}$ is given by the expression of $A$ hereabove with $V_{c}(1)=V_{c}^{0}$. Note that marginality of baroclinic instability is defined by $\left(a_{0}-d_{0}\right)^{2}+4 b_{0} c_{0}=0$. The four terms of $A_{1}$ are given by $a_{1}=0, b_{1}=V_{c}^{0}\left(1-1 /\left(2 l I_{1}(\gamma) K_{1}(\gamma)\right), c_{1}=V_{c}^{0}\left(1-\left(I_{l}(\gamma) K_{l}(\gamma)\right) /\left(I_{1}(\gamma) K_{1}(\gamma)\right)\right.\right.$ and $d_{1}=\xi V_{c}^{0}(1-$ $\left(I_{l}(\gamma) K_{l}(\gamma)\right) /\left(I_{1}(\gamma) K_{1}(\gamma)\right)$. It is easily shown that matrices $A_{0}$ and $A_{1}$ do not commute if $V_{t} \neq 0$, and therefore parametric instability is possible ([5]).

The calculations developed in [4] are adapted to our case. The contour perturbation $X$ is expanded in powers of $\varepsilon$ as $X=X_{0}+\varepsilon X_{1}+\varepsilon^{2} X_{2}+\ldots$. The fast time is $t_{0}=l b_{0} t$, slow times are defined by $t_{1}=\varepsilon t_{0}, t_{2}=\varepsilon^{2} t_{0} \ldots$

First, we assume that $h(t)=H \cos \left(\omega t_{0}\right)+\varepsilon G$. $H$ is the amplitude of the oscillatory part of the mean flow, $G$ is the supercriticality of the mean flow (if positive, or the subcriticality if negative) and $\omega$ is the pulsation of the oscillatory mean flow. This will allow parametric resonance of neutral Rossby waves, associated with the potential vorticity jump at the vortex boundary, with the oscillatory baroclinic mean flow.

To simplify the linear instability equations, we define the new variables

$$
Y_{j}=X_{j} \exp \left(i l\left(a_{0}+d_{0}\right) t_{0} / 2\right), \quad B_{0}=A_{0}+\frac{i l}{2}\left(a_{0}+d_{0}\right) I d
$$

where $I d$ is the identity matrix. The linear equations are now written

$$
\begin{gathered}
\partial_{t_{0}} Y_{0}=B_{0} Y_{0} \\
\partial_{t_{0}} Y_{1}+\partial_{t_{1}} Y_{0}=B_{0} Y_{1}+H \cos \left(\omega t_{0}\right) A_{1} Y_{0} \\
\partial_{t_{0}} Y_{2}+\partial_{t_{1}} Y_{1}+\partial_{t_{2}} Y_{0}=B_{0} Y_{2}+H \cos \left(\omega t_{0}\right) A_{1} Y_{1}+G A_{1} Y_{0}
\end{gathered}
$$


Calling $\left(y_{j t}, y_{j c}\right)$ the components of $Y_{j}$, and setting $\alpha=\left(d_{0}-a_{0}\right) /\left(2 b_{0}\right), \beta=d_{1} / b_{0}$, the equations at zeroth order in $\varepsilon$ are

$$
\begin{gathered}
\partial_{t_{0}} y_{0 t}=i \alpha y_{0 t}-i y_{0 c} \\
\partial_{t_{0}} y_{0 c}=-i \alpha y_{0 t}+i \alpha^{2} y_{0 c}
\end{gathered}
$$

By differentiating the equations in $t_{0}$, we can show that the neutral waves are described by $\partial_{t_{0}} Y_{0}=0$ and by $y_{0 c}=\alpha y_{0 t}$.

At first order, the equations are

$$
\begin{gathered}
\partial_{t_{0}} y_{1 t}+\partial_{t_{1}} y_{0 t}=i \alpha y_{1 t}-i y_{1 c}-i H \cos \left(\omega t_{0}\right) y_{0 c} \\
\partial_{t_{0}} y_{1 c}+\partial_{t_{1}} y_{0 c}=-i \alpha y_{1 t}+i \alpha^{2} y_{1 c}+i \alpha^{2} H \cos \left(\omega t_{0}\right) y_{0 t}-i \alpha \beta H \cos \left(\omega t_{0}\right) y_{0 t}
\end{gathered}
$$

We differentiate each equation in $t_{0}$ to find $\partial_{t_{0}}^{2} Y_{1}$, we make use of the fact that $\partial_{t_{0}} Y_{0}=0$, and then we integrate $\partial_{t_{0}}^{2} y_{1 t}$ to find

$$
y_{1 t}=-\frac{H}{\omega^{2}}\left[\alpha(2 \alpha-\beta) \cos \left(\omega t_{0}\right)+i \alpha \omega \sin \left(\omega t_{0}\right)\right] y_{0 t}
$$

and $y_{1 c}$ is readily obtained via the first equation of this first order system, as

$$
y_{1 c}=i \partial_{t_{1}} y_{0 t}+\frac{i H}{\omega^{2}}\left[\omega \alpha(\alpha-\beta) \sin \left(\omega t_{0}\right)+i \alpha^{2}(2 \alpha-\beta) \cos \left(\omega t_{0}\right)\right] y_{0 t}
$$

Finally, at second order in $\varepsilon$, the equations are

$$
\begin{gathered}
\partial_{t_{0}} y_{2 t}+\partial_{t_{1}} y_{1 t}+\partial_{t_{2}} y_{0 t}=i \alpha y_{2 t}-i y_{2 c}-i H \cos \left(\omega t_{0}\right) y_{1 c}-i G y_{0 c} \\
\partial_{t_{0}} y_{2 c}+\partial_{t_{1}} y_{1 c}+\partial_{t_{2}} y_{0 c}=-i \alpha y_{2 t}+i \alpha^{2} y_{2 c}+i \alpha^{2} H \cos \left(\omega t_{0}\right) y_{1 t}-i \alpha \beta H \cos \left(\omega t_{0}\right) y_{1 t}+i \alpha^{2} G Y_{0 t}-i \beta G y_{0 c}
\end{gathered}
$$

The term $\partial_{t_{2}} Y_{0}$ participates in the evolution at longer times and therefore is not kept here. The equations are time-averaged in $t_{0}$ to isolate the wave interaction with the forcing, so that all linear terms in $\cos \left(\omega t_{0}\right), \sin \left(\omega t_{0}\right)$ vanish. The value of $Y_{1}$ found above is substituted in the remaining expressions. This yields the slow-time variation of the contour, due to the interaction of neutral waves with the forcing

$$
\frac{\partial^{2} y_{0 t}}{\partial t_{1}^{2}}-\alpha^{2}(1-\chi)\left[G-\frac{\alpha^{2} H^{2}}{\omega^{2}}(1-\chi / 2)\right] y_{0 t}=0
$$

where $\chi=\beta / \alpha$. The same equation holds for $y_{0 c}$.

This equation is now physically interpreted :

For a supercritical steady flow $(G>0, H=0, \chi=0)$, adding an unsteady mean flow $(H \neq 0$ or $\chi \neq 0)$ can have several effects :

- either $\chi<1$ and the unsteady component of the flow can stabilize the vortex flow when $\alpha^{2} H^{2} / \omega^{2}>$ $G /(1-\chi / 2)$ (the case $\chi=0$ is the case described in [4]),

- or $\chi=1$, and there is linear growth of $y_{0 t}$ with the slow time $t_{1}$,

- or $\chi>1$ and stabilization will occur if $(1-\chi / 2) \alpha^{2} H^{2} / \omega^{2}<G$. Note that if $\chi>2$, this condition is automatically satisfied.

For a subcritical steady flow $(G<0, H=0, \chi=0)$, adding an unsteady mean flow $(H \neq 0$ or $\chi \neq 0)$ can have several effects :

- either $\chi<1$, and the flow remains stable in the presence of an oscillatory component of the baroclinic velocity (this includes the equal layer thickness case $\xi=0$ for which $\chi=0$; a specific study of this case is given below),

- or $\chi=1$, and there is linear growth of $y_{0 t}$ with the slow time $t_{1}$,

- or $1<\chi \leq 2$ and then the flow becomes unconditionally unstable on long times,

- or finally $2<\chi$, and the flow will be unstable on long times if $2|G|>(\chi-2) \alpha^{2} H^{2} / \omega^{2}$.

To relate $\chi$ to the vortex parameters, we write $\chi=\beta / \alpha=2 d_{1} /\left(d_{0}-a_{0}\right)=2 /(1+\phi)$, where

$$
\phi=\frac{V_{t}(1)\left(\frac{1}{l}-2 I_{l}(\gamma) K_{l}(\gamma)\right.}{V_{c}^{0} \xi\left(1-\frac{I_{l}(\gamma) K_{l}(\gamma)}{I_{1}(\gamma) K_{1}(\gamma)}\right)}
$$




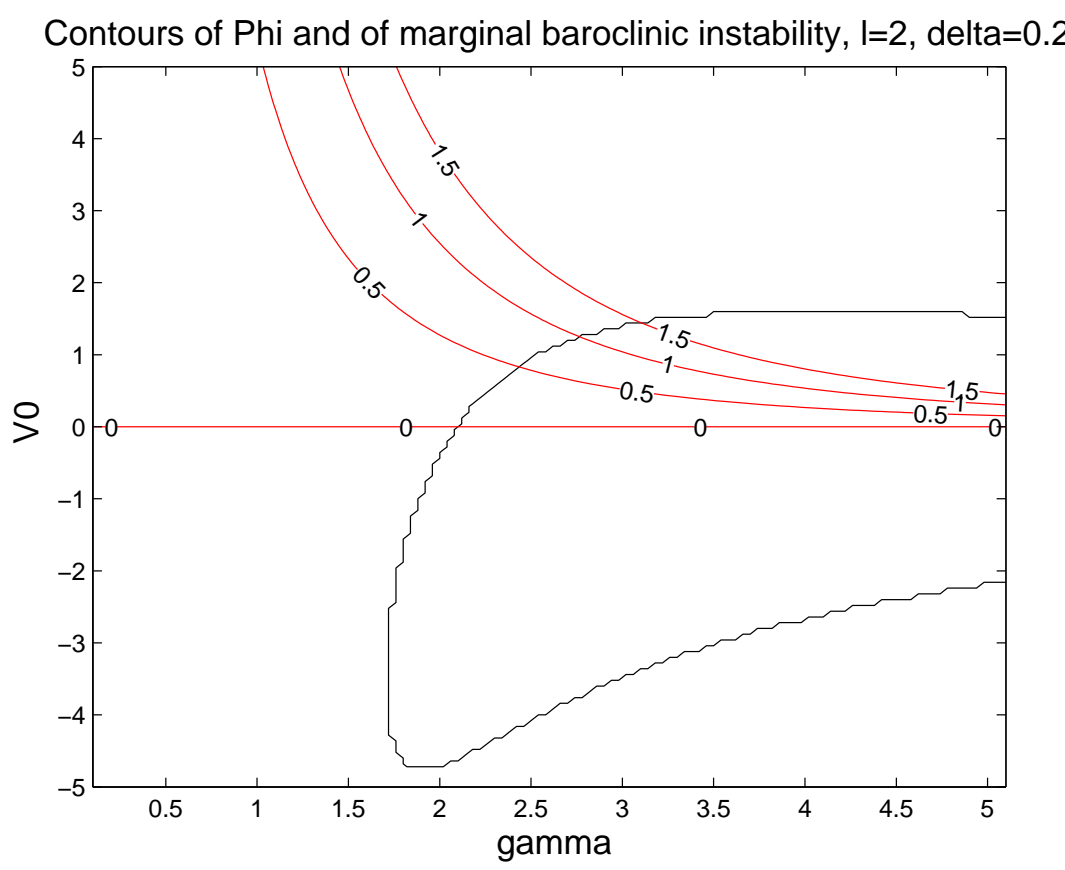

Fig. 1 Contours of $\phi$ (in red) in the $\left(\gamma, V_{0}\right)$ plane, superimposed on the marginal curve for baroclinic instability (in black).

This formulation excludes the equal layer case $\xi=0$. We can now state that the condition $\chi=1$ is $\phi=1$, and the condition $\chi=2$ is $\phi=0$. For $l=2, \delta=0.2(\xi \sim 1.8)$, the values of $\phi$ are plotted in the $\left(\gamma, V_{0}\right)$ plane (where $V_{0}=V_{t}(1) / V_{c}^{0}$, see figure 1 ). The marginal curve of baroclinic instability is superimposed. Since the isolines $\phi=0$ and $\phi=1$ cross the marginal curve, stabilization of supercritical flows and destabilization of subcritical flows can occur.

In the case of equal layer thicknesses, $\xi=0$ (and thus $\chi=0$ ); then, destabilization of a subcritical flow cannot occur with the forcing used above. But Pedlosky and Thomson ([4]) showed that a weak, low frequency forcing of the type $h(t)=\varepsilon^{2}\left(G+H \cos \left(\omega t_{1}\right)\right)$ can lead to such a destabilization. Note that $\omega t_{1}=\varepsilon \omega t_{0}$, hence the low frequency forcing. We adapt their calculation to the present case. At zeroth order in $\varepsilon$, the equations are unchanged from the previous case. At first order in $\varepsilon$, they are

$$
\begin{gathered}
\partial_{t_{0}} y_{1 t}+\partial_{t_{1}} y_{0 t}=i \alpha y_{1 t}-i y_{1 c} \\
\partial_{t_{0}} y_{1 c}+\partial_{t_{1}} y_{0 c}=-i \alpha y_{1 t}+i \alpha^{2} y_{1 c} .
\end{gathered}
$$

The same calculation as for zeroth order shows that $\partial_{t_{0}} Y_{1}=0$.

Finally, at second order, the equations are

$$
\begin{gathered}
\partial_{t_{0}} y_{2 t}+\partial_{t_{1}} y_{1 t}+\partial_{t_{2}} y_{0 t}=i \alpha y_{2 t}-i y_{2 c}-i H \cos \left(\omega t_{1}\right) y_{0 c}-i G y_{0 c} \\
\partial_{t_{0}} y_{2 c}+\partial_{t_{1}} y_{1 c}+\partial_{t_{2}} y_{0 c}=-i \alpha y_{2 t}+i \alpha^{2} y_{2 t}+i \alpha^{2} H \cos \left(\omega t_{1}\right) y_{0 t}+i \alpha^{2} G y_{0 t}
\end{gathered}
$$

Again, we exclude the term $\partial_{t_{2}} Y_{0}$ which participates in the evolution at longer times, we time-average the equations in $t_{0}$, we differentiate the first order equations in $t_{1}$ and we substitute $\partial_{t_{1}} Y_{1}$ from the second order equations. This leads to

$$
\frac{\partial^{2} y_{0 t}}{\partial t_{1}^{2}}-2 \alpha^{2}\left[G+H \cos \left(\omega t_{1}\right)\right] y_{0 t}=0
$$

This equation is a Mathieu equation which is integrated numerically. The resonant pulsation $\omega=$ $2 \sqrt{-2 \alpha^{2} G}$ is chosen. The results are shown on figure 2 for $H=0$ (non amplified oscillation) and for $H=0.1$ (parametrically amplified oscillation). Thus, contour perturbations on a baroclinically stable vortex can be amplified via resonance with a low frequency forcing. 


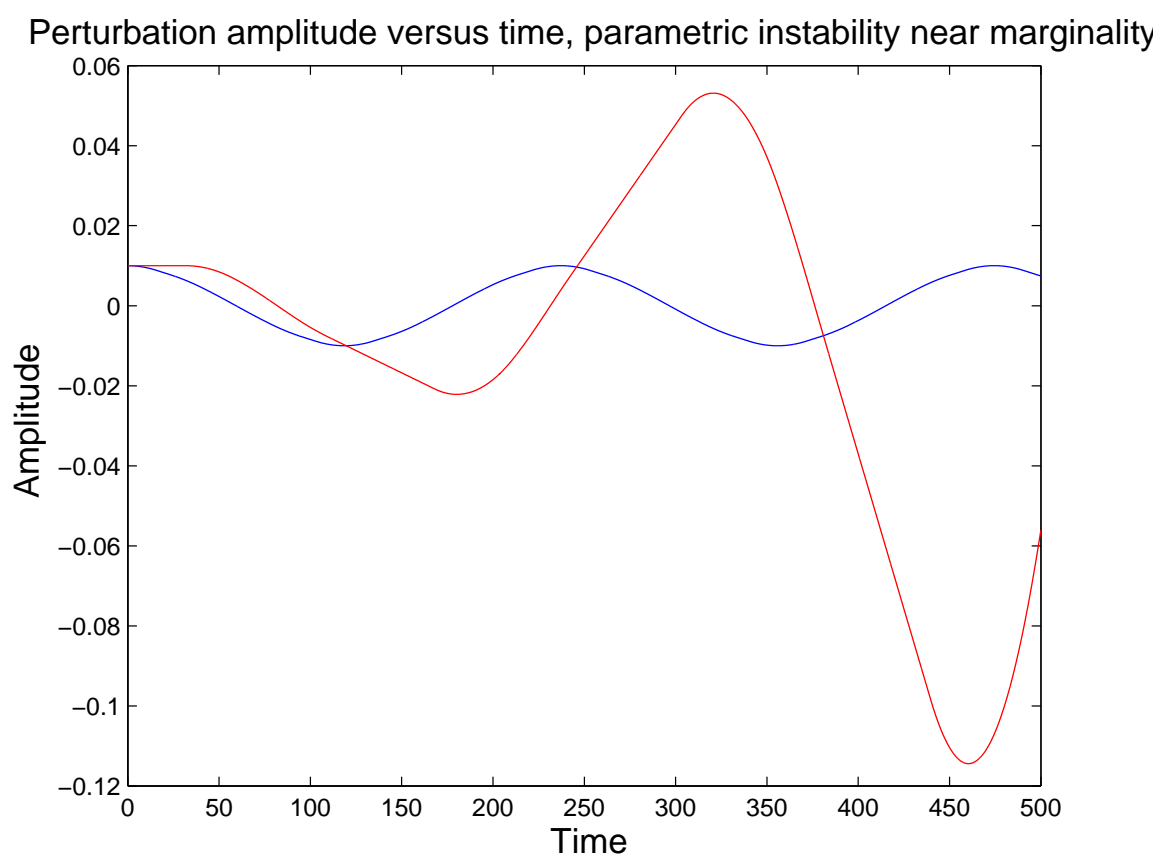

Fig. 2 Parametric amplification of the contour perturbation for $\gamma=2.0, G=-0.1, H=0.1, y_{0 t}\left(t_{1}=0\right)=$ $0.01, d y_{0 t} / d t_{1}\left(t_{1}=0\right)=0.0$ and at marginality of baroclinic instability (red curve). The steady case $H=0$ (blue curve) is shown as reference.

\section{Conclusions}

For a baroclinic vortex in a two-layer quasi-geostrophic flow, the interaction of neutral Rossby waves, associated with the vorticity jump at the vortex periphery, with the oscillatory component of the mean baroclinic velocity, can lead to parametric resonance, near marginality of baroclinic instability. Under given conditions on the steady and oscillatory mean velocities, parametric effects can stabilize vortex flows, which would otherwise be baroclinically unstable. Conversely, parametric resonance can destabilize subcritical baroclinic flows, if layer thicknesses are different. If layer thicknesses are equal, it was shown that a low frequency oscillation of the baroclinic mean flow can destabilize a subcritical vortex flow.

For application to the ocean, the present study should be extended to more complex flows.

\section{Acknowledgements}

XC acknowledges fruitful discussions with Dr Francis Poulin during the course of this work. The authors thank a referee for a thorough analysis of the paper and for fruitful suggestions.

\section{References}

1. Pedlosky, J.: The instability of continuous heton clouds. J. Atmos. Sci., 42, 1477-1486 (1985)

2. Kozlov, V.F., Makarov, V.G. and M.A. Sokolovskiy: A numerical model of baroclinic instability of axially symmetric vortices in a two-layer ocean. Izv. Atmos. Ocean. Phys., 22, 868-874 (1986)

3. Flierl, G.R.: On the instability of geostrophic vortices. J. Fluid Mech., 197, 349-388 (1988)

4. Pedlosky J. and J. Thomson: Baroclinic instability of time dependent currents J. Fluid Mech., 490, 189-215 (2003).

5. Farrell, B.F. and P.J. Ioannou: Generalized stability theory. Part II: Non-Autonomous Operators. J. Atmos. Sci., 53, 2041-2053 (1996) 